\title{
A Transient Fear Reduction by Pair-Exposure with a Non-Fearful Partner during Fear Extinction Independent from Corticosterone Level in Mice
}

\author{
Haruna Tomizawa $^{1 *}$, Daisuke Matsuzawa ${ }^{1,2}$, Shingo Matsuda ${ }^{1,3}$, Daisuke Ishii, \\ Chihiro Sutoh ${ }^{2}$, Eiji Shimizu, ${ }^{1,2}$ \\ ${ }^{1}$ Department of Cognitive Behavioral Physiology, Chiba University Graduate School of Medicine, Chiba, Japan \\ ${ }^{2}$ Research Center for Child Mental Development, Chiba University Graduate School of Medicine, Chiba, Japan \\ ${ }^{3}$ Research Fellow of the Japan Society for the Promotion of Science, Tokyo, Japan \\ Email: *haruna@graduate.chiba-u.jp
}

Received July 30, 2013; revised August 16, 2013; accepted August 21, 2013

Copyright (C) 2013 Haruna Tomizawa et al. This is an open access article distributed under the Creative Commons Attribution License, which permits unrestricted use, distribution, and reproduction in any medium, provided the original work is properly cited.

\begin{abstract}
Social interaction is known to alter behavior and emotional responses to various events. It has been reported that when fear-conditioned animals are put in a fear extinction paradigm with non-fearful conspecifics (pair-exposure), freezing behavior decreases compared to a solitary situation. However, it remains unclear whether pair-exposure during fear extinction is persistently effective in reducing the freezing response. In this study, we examined whether the effect of pair-exposure could be persistently effective on cued and contextual fear extinction. The reduction of the fear compared to the solitary condition was transiently observed only in the cued fear extinction with no difference in the subsequent recall session. We also found that the correlation between corticosterone levels and freezing behavior during extinction was disrupted in the pair-exposure situation. These results suggest that pair-exposure reduces freezing behavior in cued fear extinction, although this fear response reduction is not persistent. The pair-exposure changed an association between corticosterone levels and freezing behavior during extinction.
\end{abstract}

Keywords: Social Buffering; Pair-Exposure; Fear Extinction; Corticosterone

\section{Introduction}

Clinical exposure therapy for anxiety disorders relies on fear extinction, which is generally believed to be a form of new learning that inhibits the expression of the already acquired fear response [1]. The efficacy of the therapy is still limited to only some portion of patients [2]. Thus, recent studies have explored the methods that facilitate the consolidation of fear extinction memory and reduce fear responses in the long term [3].

Social interaction is known to alter behavior and emotional responses to various events including the fear responses in the fear extinction of the conditioned fear. Recently, Kiyokawa et al. reported that the presence of a non-fearful conspecific animal during fear extinction (pair-exposure) attenuated fear responses [4-6]. However, the question still remains whether the pair-exposure during extinction could persistently work beneficially for the

\footnotetext{
"Corresponding author.
}

consolidation of the fear extinction memory.

Little is known about the mechanism of fear attenuation by pair-exposure but presence of conspecifics has been reported to affect various hormonal levels. Although corticosterone secretion is increased by stressful events, previous studies have reported that the pair situation attenuated the increases due to exposure to a novel environment [7], a fear-inducing animal [8] and fear conditioning [9]. These phenomena are called social buffering, which can mitigate various stress responses through signals such as odor, touch and visual stimulus from conspesifics $[10,11]$. However, there has been no study that has investigated the effect of the social buffering during fear extinction on corticosterone levels. Corticosterone has been reported to enhance the excitability of amygdala neurons [12], and increases in corticosterone levels have been correlated with enhanced fear-conditioned memory in rats [13]. Thus, we hypothesized a decrease of corticosterone secretion is a factor that leads 
to the reduction of fear responses. Whereas, corticosterone affects fear memory by modulating the storage or consolidation phase [14], several other studies have reported that corticosterone treatment [15] and administration of a glucocorticoid agonist [16] facilitated fear extinction. Therefore, attenuation of corticosterone secretion possibly interrupts persistent fear reduction.

In this study, by using pavlovian cued and contextual fear conditioning paradigms, we examined whether the pair-exposure could be beneficial or not for the fear extinction learning. Our study would be a first step in determining whether pair-exposure during fear extinction might be useful in assisting the method of exposure therapy. In addition, to determine whether pair-exposure during fear extinction decreases corticosterone secretion, as in previous social buffering studies, we measured plasma corticosterone levels after fear extinction.

\section{Materials and Methods}

\subsection{Animals}

All studies involved 8 week old male C57BL/6J mice (Japan SLC, Inc, Shizuoka, Japan). To be certain mice were non-familiar, Partner group mice (same strain, sex and age) were purchased from CLEA Japan (Tokyo, Japan). There were two types of social buffering differenttially mitigate conditioned fear responses (pair-housing and pair-exposure) [5,6]. To remove the pair-housing effect, the Pair and Solitary mice were individually housed. Partner mice were housed 3 per cage. They were placed on a 12-h light/dark cycle (light on at 7 am.) with ad libitum food and water. All animals were handled for 5 days before behavioral testing. Mice were used only once. The research and animal care were carried out according to the Guide for Animal Experimentation of the Chiba University Graduate School.

\subsection{Cued Fear Conditioning, Extinction and Recall}

\subsubsection{Apparatus}

The conditioning chamber $(22.8 \times 19.7 \times 13 \mathrm{~cm}$ with a transparent wall and metal floor rods (Actimetrics, IL, USA)) was cleaned thoroughly with a $70 \%$ ethanol $/ 30 \%$ water solution before each testing. The extinction chamber was formed a polypropylene wall and an acryl floor inside conditioning chamber. The partner box was made of acrylic walls and the palisade consisted of $3 \mathrm{~mm}$ diameter stainless bars placed at $1 \mathrm{~cm}$ intervals placed into the extinction chamber. The palisade prevented physical interactions but allowed for transmission of visual, olfactory and acoustic stimulus. The extinction chamber and partner box were cleaned thoroughly with a $79.5 \%$ water $/ 19.5 \%$ ethanol $/ 1 \%$ vanilla-extract solution before each testing (Figures 1(a)-(c)).

\subsubsection{Cued Fear Conditioning}

Cued fear conditioning involves the pairing of a neutral stimulus (conditioned stimulus, CS), such as a tone with an aversive stimulus (unconditioned stimulus, US) such as an electrical footshock. After CS-US pairings, the CS presentation starts to elicit conditioned fear responses without the US. Fear extinction occurs in a process of repeated exposure to the $\mathrm{CS}$ as it decreases the fear response. In this study, mice were habituated to the conditioning chamber for $30 \mathrm{~min} \times 2$ day before fear conditioning. Mice were acclimated to the conditioning chamber for $180 \mathrm{sec}$ and then presented with six parings of a tone $(\mathrm{CS}: 3 \mathrm{kHz}, 90 \mathrm{~dB}, 30 \mathrm{sec})$ terminated with a foot shock (US: $2 \sec 0.75 \mathrm{~mA}$ ). The inter-trial interval was set as $60-180 \mathrm{sec}$. After the last CS-US parings, mice were returned to the home cage following $30 \mathrm{sec}$ no-stimulus period. After matching for equivalent levels of freezing during last $\mathrm{CS}$, conditioned mice were divided into 2 groups; Pair $(\mathrm{n}=17)$ and Solitary group $(\mathrm{n}=$ 17).

\subsubsection{Extinction and Recall}

Twenty-four hours after fear conditioning, conditioned mice were acclimated to the extinction chamber for 180 sec and exposed to the CS 40 times with a partner separated by palisade (Pair group) or alone (Solitary group).

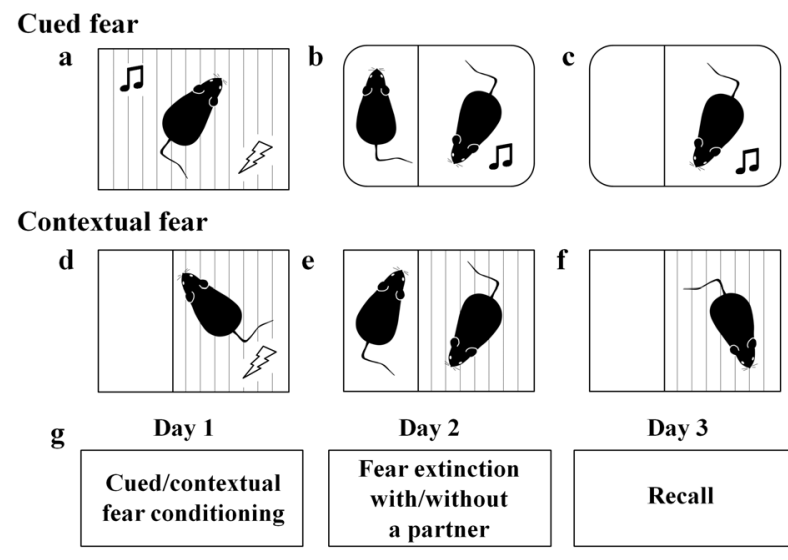

Figure 1. Experimental apparatus and design. (a)-(c) In the cued fear experiment, the chamber was divided by palisade for a test animal and a partner during the extinction and Recall. Extinction for the Pair group was performed with a partner mouse placed into the partner box, whereas extinction for the Solitary group was performed with an empty partner box (b); The Recall was performed alone by both groups (c); (d)-(f) In the contextual fear experiment, the partner box always placed into the chamber. The conditioning and Recall were performed with an empty partner box ((d), (f)). Extinction for the Pair group was performed with a partner mouse placed into the partner box, whereas extinction for the Solitary group was performed with an empty partner box (e); (g) Time schedule of the experiment. 
The inter-trial interval was set as 5 sec. Partner mice were non-familiar and non-shocked according to previous studies [5,6]. Partner mice were habituated to the partner box for $30 \mathrm{~min} \times 2$ days before extinction. To investigate the persistent effect of pair-exposure, the Recall was performed 24 hours after fear extinction. Pair and Solitary group were acclimated for $180 \mathrm{sec}$ in the extinction chamber and exposed the CS 10 times alone and freezing behavior was measured first $5 \mathrm{CSs}$. The inter-trial interval was set as $5 \mathrm{sec}$.

\subsection{Contextual Fear Conditioning, Extinction and Recall}

\subsubsection{Apparatus}

The conditioning chamber and the partner box were identical to those of cued fear experiments. To avoid the change of context, the partner box was always placed into the conditioning chamber. The conditioning chamber and the partner box were cleaned thoroughly with $70 \%$ ethanol/30\% water solution before each testing (Figures 1(d)-(f)).

\subsubsection{Contextual Fear Conditioning}

In contextual fear conditioning, fear is conditioned with a context, a conditioning chamber, where mice receive electrical foot shocks as US. In this study, Mice were habituated to the conditioning chamber for $15 \mathrm{~min}$ twice $\times 1$ day before fear conditioning. Mice were acclimated for $180 \mathrm{sec}$ and conditioned to six foot shocks (US: $2 \mathrm{sec}$ $0.75 \mathrm{~mA})$. These shock timings were the same as cued fear conditioning without tones. After the last shock, mice were returned to home cage following $180 \mathrm{sec}$ no-stimulus period. After matching for equivalent levels of freezing during last $180 \mathrm{sec}$, conditioned mice were divided into 2 groups; Pair $(\mathrm{n}=14)$ and Solitary group ( $=14)$.

\subsubsection{Extinction and Recall}

Twenty-four hours after the fear conditioning, conditioned mice were exposed to conditioning chamber with a partner separated by palisade (Pair group) or alone (Solitary group). Partner mice were non-familiar and non-shocked as well as cued fear extinction. The partner mice were habituated to the partner box for $30 \mathrm{~min} \times 2$ days before extinction. To investigate the persistent effect of pair-exposure, the Recall was performed 24 hours after fear extinction. Pair and Solitary group were exposed to the conditioning chamber for $10 \mathrm{~min}$ alone and freezing behavior was measured first $5 \mathrm{~min}$.

\subsection{Hormone Analysis}

Cued fear conditioning and extinction were done in the same way described above. Thirty $(n=22$ in Pair and 24 in Solitary) and $60 \min (\mathrm{n}=11$ in Pair and 12 in Solitary) after the introduction of cued fear extinction, Pair and Solitary mice were anesthetized with pentobarbital sodium. These time points covered a timing of corticosterone reduction in previous reports [7-9]. Their blood was collected in heparinized tubes immediately after anesthesia as soon as possible. These collection performed between 9:00 and 13:00. The collected blood was centrifuged at $5000 \mathrm{rpm}$ for $15 \mathrm{~min}$ at $4^{\circ} \mathrm{C}$, and the plasma was stored at $-80^{\circ} \mathrm{C}$ until analysis. Plasma corticosterone levels were determined using the Enzyme Immunoassay Kit (Assay Designs, Inc., MI, USA) according to the manufacturer's instructions.

\subsection{Measurement and Statistical Analysis}

Activity of mice was monitored by FreezeFrame (Actimetrics Software, 1621 Elmwood Ave Wilmette IL 60091, USA). Freezing (no visible movement except respiration) was scored every $30 \mathrm{sec}$ and converted to a percentage [(freezing observations/total observations) $\times 100]$. Data of fear extinction were analyzed using twoway repeated measures of ANOVA. Bonferroni's correction was used for post hoc comparisons. Data of Recall and plasma corticosterone concentrations were analyzed using student's t-test. Associations of hormonal level and freezing behavior were examined by Pearson correlation. Values of $p<0.05$ were considered as statistically significant. All analyses were conducted with the software SPSS 12.0 for Windows (SPSS, Chicago, Illinois). Data are shown as mean $\pm \mathrm{SEM}$ for all results.

\section{Results}

\subsection{Pair-Exposure Effect on Freezing Response in Cued Fear Extinction and Recall}

In the cued fear extinction, a two-way repeated measures of ANOVA showed significant main effects of trials $(F(5.149)=2.704, p<0.001)$ and group $(F(1.32)=$ $10.075, p<0.01)$ and significant interaction between group $\times$ trials $(F(5,149)=2.704, p<0.05)$. Bonferroni's correction showed that freezing responses of Pair mice significantly lower than those of Solitary mice during the Trial 1, $5(p<0.05)$ and Trial 2, $6(p<0.01)$ (Figure 2(a)). To investigate the persistent effect of pair-exposure, we conducted Recall 24 hours after extinction. In the Recall, student's $t$-test showed no significant difference in the two group ( $t(32)=-1.022)$ (Figure 2(a)). These findings indicated that pair-exposure during cued fear extinction reduced freezing behavior, but did not ameliorate persistent fear responses.

\subsection{Pair-Exposure Effect on Freezing Response in Contextual Fear Extinction and Recall}

In the contextual fear extinction, a two-way repeated 


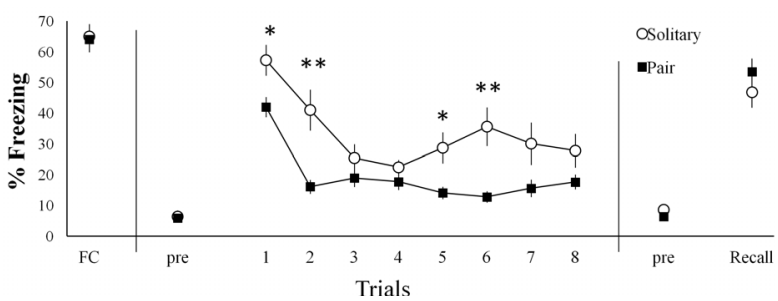

(a)

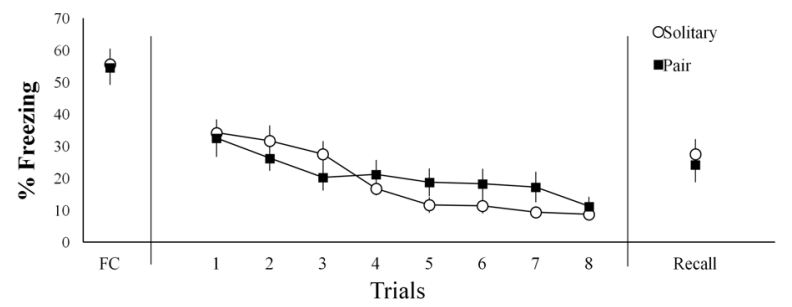

(b)

Figure 2. Effect of pair-exposure on fear responses. (a) In cued fear extinction, the Pair group exhibited a significant reduction of freezing responses compared to the Solitary group $\left({ }^{*} p<0.05\right.$ and $\left.{ }^{* *} p<0.01\right)$, whereas there was no difference of freezing response between the two groups in the Recall. (b) In the contextual fear extinction, there was no effect of pair-exposure on contextual fear responses. In the cued fear conditioning (FC), data were shown as \% freezing during last $\mathrm{CS}$, and in the extinction and recall, data were showed average of $\%$ freezing every 5 CSs $(n=17 /$ group $)$ (a); In the contextual fear conditioning (FC), data were shown as \% freezing during post 180 sec from last shock, and in the extinction and recall, data were shown as average of $\%$ freezing every $5 \mathrm{~min}(\mathrm{n}=14 /$ group) (b).

measures of ANOVA showed significant main effects of trials $(F(4.107)=25.897, p<0.001)$ and significant interaction between group $\times$ trials $(F(4.107)=3.364, p<$ $0.05)$, but did not show significant main effect of group $(F(1.26)=0.163)$ (Figure 2(b)). Bonferroni's correction did not show any significant differences in freezing responses between Pair and Solitary group. Twenty-four hours after the fear extinction, the Pair and Solitary mice were exposed to the conditioning chamber alone. In the Recall, student's $t$-test showed no significant differences in group $(t(26)=0.441)$ (Figure 2(b)). These results indicated that pair-exposure during contextual fear extinction did not attenuate the freezing response. The pairexposure induced reduction in freezing occurred specifically during cued fear extinction.

\subsection{Association between Corticosterone Levels and \% Freezing in the Fear Extinction}

The presence of conspecifics during various stressful events has been reported to attenuate the increase in corticosterone secretion [7-9]. However, there has been no study that has investigated the effect of pair-exposure during fear extinction on corticosterone levels. To deter- mine whether pair-exposure attenuates corticosterone secretion, we sampled plasma from the Solitary and Pair group 30 and 60 min after the introduction of cued fear extinction. We focused on cued fear extinction because reduction of fear responses appeared only in the cued fear extinction. A two-way repeated measures of ANOVA showed significant main effect of trials $(F(5.365)=$ $34.284, p<0.001)$ and group $(F(1.68)=17.684, p<$ $0.001)$, but did not show significant interaction between group $\times$ trials $(F(5.365)=1.904)$. Student's $t$-test showed no significant difference in group on plasma corticosterone levels $30 \mathrm{~min}(t(44)=1.100$, Figure 3(a)) and 60 min after extinction $(t(21)=-0.093$, Figure 3(b)). These results indicated that pair-exposure during fear extinction did not decrease corticosterone secretion. Pearson's correlation showed a significant positive correlation between freezing levels (during the last $10 \mathrm{CS}$ exposures) and corticosterone levels $30 \min (R(24)=0.515, p<0.01)$ and $60 \min (R(12)=0.665, p<0.05)$ after extinction for the Solitary group (Figures 3(c) and (d)). However, these effects were not present in the Pair group $(R(22)=$ -0.270 and $R(11)=-0.388$, respectively). These findings suggest that corticosterone levels after extinction in Solitary group would predict freezing responses during extinction, but corticosterone levels in Pair group would not.

\section{Discussion}

In the current study, we examined whether or not the pair-exposure could be beneficial for the extinction learning on fear responses, and corticosterone levels after the fear extinction. In brief, in the cued fear extinction paradigm, we found that pair exposure resulted in the accelerated reduction of the freezing behavior compared to the solitary group. However, the effect was transient, the two groups did not show significant difference in $\%$ freezing in the Recall. In the contextual fear extinction paradigm, the pair-exposure effect on freezing behavior was not observed with non-fearful partner during the fear extinction. A positive correlation between freezing levels during extinction and plasma corticosterone levels after cued fear extinction was observed in the solitary, but not in the pair-exposed group.

In this study, the presence of a partner reduced freezing responses during cued fear extinction. As described above, the pair-exposure reduced the fear response in fear extinction only transiently, which resulted in no difference in the freezing behavior with solitary group in the Recall. The results suggest that pair-exposure facilitated acquisition but did not alternate the consolidation of fear extinction memories. Clinically, presence of another person or therapists during extinction would be a safety signal which might lead to interfere with extinction learning. Such safety signal could be a conditioned in- 
a

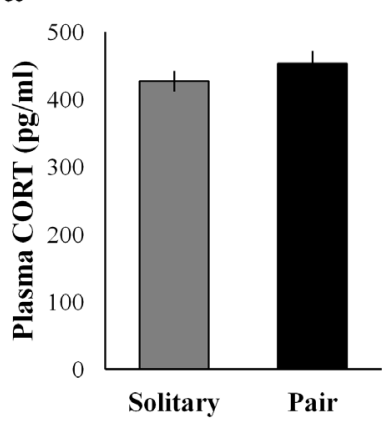

b

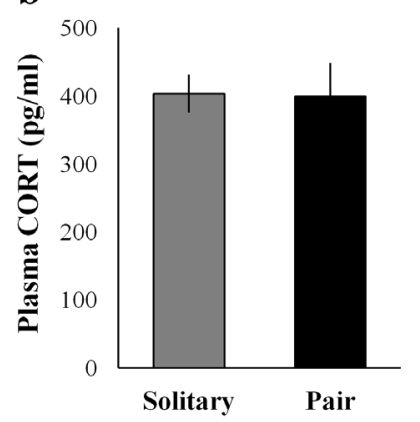

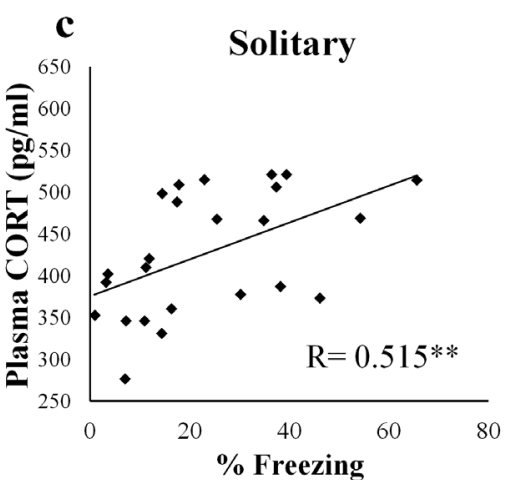

d

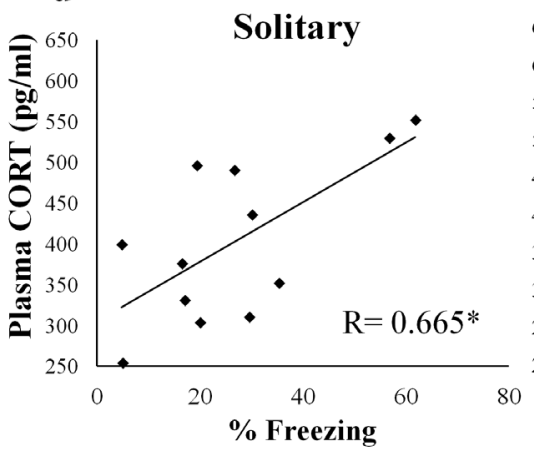

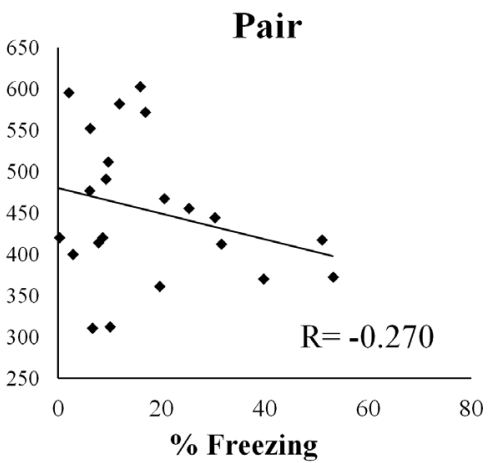

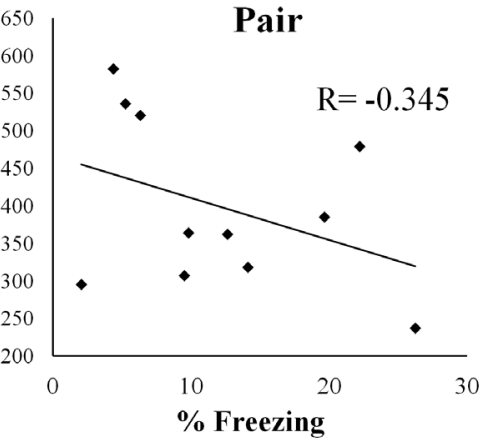

Figure 3. Plasma corticosterone levels after pair-exposure in cued fear extinction. (a) Plasma corticosterone levels 30 min after extinction were similar in each group; (b) Plasma corticosterone levels 60 min after extinction were similar in each group; (c) Plasma corticosterone levels $\mathbf{3 0}$ min after extinction and freezing responses were significantly correlated in the Solitary group $\left({ }^{* *} p<0.01\right)$; whereas the correlation was not observed in the Pair group; (d) Plasma corticosterone levels 60 min after extinction and freezing responses were significantly correlated in the Solitary group $\left({ }^{*} p<0.05\right)$, whereas the correlation was not observed in the Pair group.

hibitor which would alleviate conditioned response (CR) in the short term but maintain the $\mathrm{CR}$ when subsequently tested with conditioned stimuli without the signal [3]. In our experiments, existing of conspecific animal neither enhance the consolidation of fear extinction, nor worsen the fear response as a conditioned inhibitor in both cued and contextual situations. In the usual exposure therapy, patients need to overcome the initial fear and anxiety provoked in the first exposure. Thus, to apply our results to clinical exposure therapy for anxiety disorders, the social interaction would be beneficial as an easy induction for patients by reducing initial fear response without interfering fear extinction learning in the subsequent trials.

Interestingly, the reduction of freezing response was observed in the cued fear extinction, but not in the contextual fear extinction, which suggests the pair-exposure effect could be different depending on the fear extinction paradigm. It could be expected that pair-exposure would attenuate the fear responses of contextual conditioned animals because there is a possibility that the contextual CS was disrupted by the partner [5]. However, the pair-exposure in the contextual fear extinction did not affect freezing responses in our study which means the pair-exposure did not interfere with the fear extinction in the normal condition. Guzman et al. has also reported that the influences of exposure to conspecifics differ between cued and contextual paradigms [17]. The pre-exposure to non-fearful conspecifics before fear conditioning produced a persistent reduction in fear responses during contextual fear extinction, whereas this pre-exposure was not effective in cued fear response [17]. Although, future study is needed to clarify the dissociative effect of pair exposure, methodological factor might have affected the results. In our study, mice were separated by the palisade which prevented physical interactions but allowed for transmission of visual, olfactory and acoustic stimuli. Olfactory signal has been reported as an essential factor for stress reduction by conspecifics [6]. Olfactory signals were transmitted to the amygdala through the posteromedial region of the olfactory peduncle, and mitigated activity of the amygdala [18]. Thus, combined with our results, the presence of a non-fearful partner's odor during CS exposure may be necessary for pair-exposure effects on cued fear extinction. The contextual fear conditioning requires both the hippocampus and the amygdala, whereas cued fear conditioning does not require the hippocampus [19]. The primary olfactory cor- 
tex projects not only to the amygdala, but also to the entorhinal cortex, which in turn projects to the hippocampus [20]. The hippocampus might interfere with the mitigation of the amygdala activity by the olfactory signal in the contextual paradigm.

It should be noted that the palisade prevented the physical interactions between the two animals. This is an apparent difference between our study and the previous study in which attenuated fear response was reported when the animal (rat) was re-exposed to the shock context with a partner after contextual fear conditioning [4]. Although we did not examine the behavioral testing without palisade due to the limited ability of our analysis software, several studies have suggested the importance of physical contact with a partner in social buffering. For example, the prolactin response to open fields is attenuated by play with conspecifics in juvenile rats, and this attenuation was not observed when the pair was separated by a partition [21]. Pair-housing decreased anxiety behavior in the elevated plus maze, and full physical contact is necessary for the emergence of the buffering effect of pair housing on social stress [22].

The overall corticosterone levels were not different between the two groups, and a positive correlation between corticosterone levels and freezing responses in the Solitary group was observed in agreement with previous studies [13]. Corticosterone is believed to enhance memory for emotionally arousing information [23] and enhance the excitability of amygdala neurons [12]. Thus, the correlation between corticosterone and freezing responses in the Solitary group likely reflects the intensity of fear memories. In the Pair group, however, this correlation was not observed. The effect of social buffering on corticosterone response during stressful events is actually inconsistent among studies. Some reported that social buffering prevented increase of corticosterone during stressful events [7-9], whereas there are reports that social buffering does not decrease corticosterone responses to novel environments or fear inducing animal [24,25]. It cannot be denied these differences in the effects of the social buffering may be attributable to differences in species, strains, or environments, our findings suggest that the effect of the social buffering on behavioral response could be independent from the corticosterone response, which would not affect the consolidation of extinction learning.

\section{Conclusion}

Our data showed that pair-exposure reduced freezing behavior in cued fear extinction, but not in contextual fear extinction. This fear response reduction was transient and the subsequent fear response was observed normally. Corticosterone level after the fear extinction was correlated with \% freezing in the Solitary condition but not in the Paired condition, which suggested that the mechanism under the effect of social buffering might be distinct from the regulation of the stress hormone.

\section{Acknowledgements}

This work was supported by JSPS KAKENHI Grant Number 24791196.

\section{REFERENCES}

[1] K. M. Myers and M. Davis, "Mechanisms of Fear Extinction," Molecular Psychiatry, Vol. 12, No. 2, 2007, pp. 120-150. doi:10.1038/sj.mp.4001939

[2] R. Bradley, J. Greene, E. Russ, L. Dutra and D. Westen, "A Multidimensional Meta-Analysis of Psychotherapy for PTSD," American Journal of Psychiatry, Vol. 162, No. 2, 2005, pp. 214-227. doi:10.1176/appi.ajp.162.2.214

[3] M. G. Craske, K. Kircanski, M. Zelikowsky, J. Mystkowski, N. Chowdhury and A. Baker, "Optimizing Inhibitory Learning during Exposure Therapy," Behaviour Research and Therapy, Vol. 46, No. 1, 2008, pp. 5-27. doi:10.1016/j.brat.2007.10.003

[4] Y. Kiyokawa, T. Kikusui, Y. Takeuchi and Y. Mori, "Partner's Stress Status Influences Social Buffering Effects in Rats," Behavioral Neuroscience, Vol. 118, No. 4, 2004, pp. 798-804. doi:10.1037/0735-7044.118.4.798

[5] Y. Kiyokawa, Y. Takeuchi and Y. Mori, "Two Types of Social Buffering Differentially Mitigate Conditioned Fear Responses," European Journal of Neuroscience, Vol. 26, No. 12, 2007, pp. 3606-3613. doi:10.1111/j.1460-9568.2007.05969.x

[6] Y. Kiyokawa, Y. Takeuchi, M. Nishihara and Y. Mori, "Main Olfactory System Mediates Social Buffering of Conditioned Fear Responses in Male Rats," European Journal of Neuroscience, Vol. 29, No. 4, 2009, pp. 777785.

[7] S. E. File and L. A. Peet, "The Sensitivity of the Rat Corticosterone Response to Environmental Manipulations and to Chronic Chlordiazepoxide Treatment," Physiology \& Behavior, Vol. 25, No. 5, 1980, pp. 753-758. doi:10.1016/0031-9384(80)90379-0

[8] J. L. Vogt, C. L. Coe and S. Levine, "Behavioral and Adrenocorticoid Responsiveness of Squirrel Monkeys to a Live Snake: Is Flight Necessarily Stressful?" Behavioral and Neural Biology, Vol. 32, No. 4, 1981, pp. 391405. doi:10.1016/S0163-1047(81)90826-8

[9] M. E. Stanton, J. M. Patterson and S. Levine, "Social Influences on Conditioned Cortisol Secretion in the Squirrel Monkey," Psychoneuroendocrinology, Vol. 10, No. 2, 1985, pp. 125-134.

doi:10.1016/0306-4530(85)90050-2

[10] M. B. Hennessy, S. Kaiser and N. Sachser, "Social Buffering of the Stress Response: Diversity, Mechanisms, and Functions," Frontiers in Neuroendocrinology, Vol. 30, No. 4, 2009, pp. 470-482.

doi:10.1016/j.yfrne.2009.06.001

[11] T. Kikusui, J. T. Winslow and Y. Mori, "Social Buffering: 
Relief from Stress and Anxiety," Philosophical Transactions of the Royal Society B, Vol. 361, No. 1476, 2006, pp. 2215-2228. doi:10.1098/rstb.2006.1941

[12] S. Duvarci and D. Pare, "Glucocorticoids Enhance the Excitability of Principal Basolateral Amygdala Neurons," The Journal of Neuroscience, Vol. 27, No. 16, 2007, pp. 4482-4491. doi:10.1523/JNEUROSCI.0680-07.2007

[13] M. I. Cordero, J. J. Merino and C. Sandi, "Correlational Relationship between Shock Intensity and Corticosterone Secretion on the Establishment and Subsequent Expression of Contextual Fear Conditioning," Behavioral Neuroscience, Vol. 112, No. 4, 1998, pp. 885-891. doi:10.1037/0735-7044.112.4.885

[14] F. Bermúdez-Rattoni, "Neural Plasticity and Memory: From Genes to Brain Imaging," CRC Press, Boca Raton, 2007. doi:10.1201/9781420008418

[15] R. Zeitlin, S. Patel, R. Solomon, J. Tran, E. J. Weeber and V. Echeverria, "Cotinine Enhances the Extinction of Contextual Fear Memory and Reduces Anxiety after Fear Conditioning," Behavioural Brain Research, Vol. 228, No. 2, 2012, pp. 284-293. doi:10.1016/j.bbr.2011.11.023

[16] Y. L. Yang, P. K. Chao and K. T. Lu, "Systemic and Intra-Amygdala Administration of Glucocorticoid Agonist and Antagonist Modulate Extinction of Conditioned Fear," Neuropsychopharmacology, Vol. 31, 2006, pp. 912-924. doi:10.1038/sj.npp.1300899

[17] Y. F. Guzman, N. C. Tronson, A. Guedea, K. H. Huh, C. Gao and J. Radulovic, "Social Modeling of Conditioned Fear in Mice by Non-Fearful Conspecifics," Behavioural Brain Research, Vol. 201, No. 1, 2009, pp. 173-178. doi:10.1016/j.bbr.2009.02.024

[18] Y. Kiyokawa, Y. Wakabayashi, Y. Takeuchi and Y. Mori, "The Neural Pathway Underlying Social Buffering of Conditioned Fear Responses in Male Rats," European Journal of Neuroscience, Vol. 36, No. 10, 2012, pp. 3429-3437. doi:10.1111/j.1460-9568.2012.08257.x
[19] R. G. Phillips and J. E. LeDoux, "Differential Contribution of Amygdala and Hippocampus to Cued and Contextual Fear Conditioning," Behavioral Neuroscience, Vol. 106, No. 2, 1992, pp. 274-285. doi:10.1037/0735-7044.106.2.274

[20] P. M. Lledo, G. Gheusi and J. D. Vincent, "Information Processing in the Mammalian Olfactory System," Physiological Reviews, Vol. 85, No. 1, 2005, pp. 281-317. doi:10.1152/physrev.00008.2004

[21] J. H. Wilson, "Prolactin in Rats Is Attenuated by Conspecific Touch in a Novel Environment," Cognitive, Affective, \& Behavioral Neuroscience, Vol. 1, No. 2, 2001, pp. 199-205. doi:10.3758/CABN.1.2.199

[22] T. Nakayasu and K. Kato, "Is Full Physical Contact Necessary for Buffering Effects of Pair Housing on Social Stress in Rats?" Behavioural Processes, Vol. 86, No. 2, 2011, pp. 230-235. doi:10.1016/j.beproc.2010.12.002

[23] T. W. Buchanan and W. R. Lovallo, "Enhanced Memory for Emotional Material Following Stress-Level Cortisol Treatment in Humans," Psychoneuroendocrinology, Vol. 26, No. 3, 2001, pp. 307-317. doi:10.1016/S0306-4530(00)00058-5

[24] A. Armario, G. Luna and J. Balasch, "The Effect of Conspecifics on Corticoadrenal Response of Rats to a Novel Environment," Behavioral and Neural Biology, Vol. 37, No. 2, 1983, pp. 332-337. doi:10.1016/S0163-1047(83)91425-5

[25] C. L. Coe, D. Franklin, E. R. Smith and S. Levine, "Hormonal Responses Accompanying Fear and Agitation in the Squirrel Monkey," Physiology \& Behavior, Vol. 29, No. 6, 1982, pp. 1051-1057. doi:10.1016/0031-9384(82)90297-9 